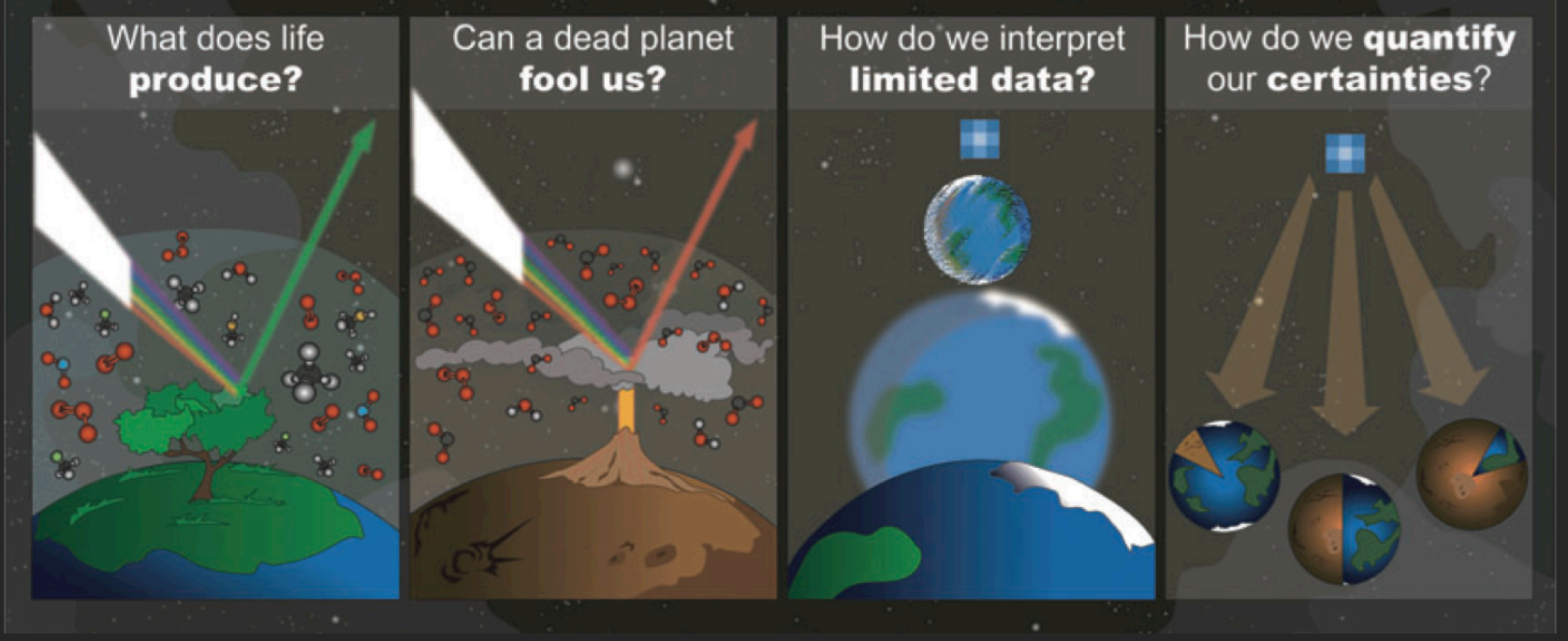

\title{
Synergies between exoplanet and Solar System life detection efforts: Encouraging collaboration to enhance science return
}

Primary authors:

Niki Parenteau (NASA Ames Research Center, mary.n.parenteau@nasa.gov 650.604.0784)

Shawn Domagal-Goldman (NASA Goddard Space Flight Center, shawn.goldman@nasa.gov 301.614.6245)

Nancy Y. Kiang (NASA Goddard Institute for Space Studies, nancy.y.kiang@nasa.gov 212.678.5553)

Co-authors:

Edward Schwieterman (Univ. of California, Riverside, eschwiet@ucr.edu); Victoria Meadows (Univ. of Washington, meadows@uw.edu); Christopher T. Reinhard (Georgia Institute of Technology, chris.reinhard@eas.gatech.edu); Hilairy E. Hartnett (Arizona State University, h.hartnett@asu.edu); Timothy Lyons (Univ. of California, Riverside, timothyl@ucr.edu); Joshua Krissansen-Totton (Univ. of California, Santa Cruz, jkt@ucsc.edu); David Catling (Univ. of Washington, dcatling@uw.edu); Yuka Fujii (National Astronomical Observatory of Japan (NAOJ), yuka.fujii.ebihara@gmail.com)

Co-signers:

Giada Arney, NASA GSFC; Monica Vidaurri, Howard University/NASA GSFC; Ravi Kopparapu, NASA GSFC; Owen Lehmer, NASA ARC; Timothy Holt, University of Southern Queensland; Chester 'Sonny' Harman, NASA ARC; David J. Des Marais, NASA ARC; Dawn Gelino, NASA Exoplanet Science Institute, IPAC/Caltech; Graham Lau, Blue Marble Space Institute of Science (BMSIS); Svetlana Shkolyar, USRA/NASA GSFC/BMSIS; Tori Hoehler, NASA ARC; Wesley D. Swingley, Northern Illinois University; Luoth Chou, NASA GSFC/ Georgetown University; Brandi Kiel Reese, Dauphin Island Sea Lab; Natasha Batalha, NASA ARC; Vinayak Shastri, University of St Andrews; Anneliese Ardizzone, NASA GSFC; Jaime Crouse, NASA GSFC; Milena Popovic, BMSIS/NASA ARC; Andro Rios, BMSIS/NASA ARC; Christopher Dateo, NASA ARC; Chuhong Mai, Arizona State University; Evan L. Sneed, Pennsy/vania State University; Britney Schmidt, Georgia Institute of Technology, Dragos G. Zaharescu, University of Wisconsin Green Bay. 


\section{Executive summary:}

The rapid advance of exoplanet discovery, planetary systems science, and telescope technology will soon allow scientists to search for life beyond our Solar System through direct observation of extrasolar planets. This endeavor will occur alongside searches for habitable environments and signs of life within our Solar System. These searchers will require separate observational techniques, and exoplanets pose an additional challenge of having relatively limited data on any individual world. However, these searches are thematically related and will inform each other. This white paper will explore those synergies, and encourage an exchange of "lessons learned" between the Solar System and exoplanet communities, in particular focusing on quantitative frameworks for interpretation of biosignatures. Enhanced communication across the NASA Science Mission Directorate (in particular between the Astrophysics and Planetary Science Divisions) will prevent stove-piping of data and evaluation protocols, and will enhance the science return and bolster the theoretical and empirical foundation to interpret life detection mission results.

\section{Key recommendations:}

- Hold workshops to educate and socialize exoplanet and planetary communities to their different lineages of biosignature research and life detection approaches.

- Increase communication and coordination between Astrophysics and Planetary Science Divisions and ROSES R\&A funding opportunities, especially as related to funding exoplanet research within Planetary programs.

- Host more cross-divisional meetings between the different analysis groups (AGs): Mars Exploration Program Analysis Group (MEPAG), Outer Planets Assessment Group (OPAG), Small Bodies Assessment Group (SBAG), and Exoplanet Exploration Program Analysis Group (ExoPAG).

- Establish "exoplanet participating scientist" or "planetary participating scientist" positions in Solar System and exoplanet life detection missions, respectively (Arney et al., 2020; Marley et al., 2020).

- Amplify and reinforce recommendations in other exoplanet-Solar System synergy white papers:

- Please refer to the Arney et al. (2020) white paper "Exoplanets in our Backyard" for a report on the scientific and programmatic findings from the recent joint exoplanet-planetary workshop, which shows how NASA and the science community can encourage and nurture research at the intersection of the Solar System and exoplanet fields.

- Please refer to the Marley et al. (2020) white paper "Enabling Effective Exoplanet/Planetary Collaborative Science" for specific structural and policy recommendations.

- Please refer to the Schmidt et al. (2020) white paper on "Enabling Progress Towards Life Detection on NASA Missions: A White Paper from the Network for Life Detection" and the Hoehler et al. (2020) white paper "Groundwork for Life Detection," which advocate that community-level efforts be pursued to establish a standardized, evaluative framework that supports apples-to-apples assessment of diverse biosignatures' utility in addressing life detection objectives. 


\section{Introduction}

This white paper summarizes products from the Exoplanet Biosignatures Workshop Without Walls (EBWWW), then compares and contrasts life detection efforts for exoplanet and Solar System targets and provides key recommendations for an exchange of "lessons learned" to enhance science return on life detection missions. This white paper also serves to update the planetary science community on the state of the exoplanet biosignatures field.

The EBWWW was composed of a series of online and in-person activities, with participation from the international exoplanet and astrobiology communities, to assess the state of the science and future research needs for the remote detection of life on planets outside our Solar System. These activities culminated in five journal articles that respectively cover: 1) a review of known and proposed biosignatures (Schwieterman et al., 2018), 2) a review of $\mathrm{O}_{2}$ as a biosignature as an end-to-end example of the contextual knowledge required to rigorously assess any claims of life on exoplanets (Meadows et al., 2018); 3) a generalized statistical approach to place qualitative understanding and available data in a formal quantitative framework according to current understanding (Catling et al., 2018); 4) identification of needs to advance that statistical framework, and to develop or incorporate other conceptual frameworks for biosignature assessment (Walker et al., 2018), and 5) a review of the upcoming observatories - both planned and possible - that could provide the data needed to search for exoplanet biosignatures (Fujii et al., 2018). These articles were written by an interdisciplinary and international community of scientists, incorporating input from both an open public comment period and an anonymous journal peer review process. As such, they represent the community-wide scientific consensus on the state of the field, and on the research priorities to further the search for life on exoplanets. These articles were submitted as the final report of the ExoPAG's 16th Science Analysis Group. However, this summary was not reviewed by that group and should not be considered as being endorsed explicitly by ExoPAG.

\section{Progress Since the 2010 New Worlds, New Horizons Decadal Survey}

Expanding the library of signs of life. Analyses of a planet's spectrum, even from a single spatial element, can yield information on the presence or absence of chemicals that absorb specific wavelengths of light. It is this limited information upon which many of our proposed biosignatures, as well as other features of the planet's environmental context, must be identified. Much of the history of remote detection of biosignatures focused on spectral features of specific biological byproducts or global phenomena resulting from life. A review of exoplanet biosignatures is presented in Schwieterman et al. (2018), updating a prior review by Des Marais et al. (2002), which was considered in the New Worlds, New Horizons decadal survey. There have been three major developments in exoplanet biosignature science since 2010: the generation of a broader list of potential biosignatures, more comprehensive simulations of these signatures in the context of planetary environments, and consideration of abiotic means through which these signatures could be generated on both living and nonliving worlds.

Novel candidate biosignatures. There has been a large expansion in the catalog of proposed biosignatures for the community to consider. For photosynthetic pigments, organisms that extend the wavelengths of light that can drive oxygenic photosynthesis have been discovered (Ho et al. 2016; Li et al., 2015), increasing the types of star-planet combinations that can sustain this metabolism (Takizawa et al., 2017). Surface pigments other than those used for oxygenic photosynthesis have also been proposed, including bacteriorhodopsin and other pigments (e.g., Schwieterman et al., 2015a, Hegde et al., 2015). For atmospheric biosignatures, several thousand 
volatile gases have been identified as worthy of further consideration (Seager et al., 2016). On planets lacking oxygen, atmospheric features such as organic hazes have also been identified as possible signs of life (Arney et al., 2016). Sustained efforts to rigorously describe the new wealth of biosignature features are critically needed.

3D simulation of living worlds. Modeling tools have become critical in simulating biosignatures on a global scale. These include photochemical and climate models that can selfconsistently simulate these biosignatures within their planetary context. A significant advance in this area since 2010 is the utilization of 3-dimensional (3D) spectral models (e.g., Robinson et al., 2011; Schwieterman et al., 2015b). 3D general circulation models (GCMs) are emerging as important theoretical tools to explore the dynamics of planetary climates and to expand conceptualization of the habitable zone (e.g., Turbet et al.2016; Way et al., 2017). Further development of these modeling capabilities will be needed to apply coupled biosphere-atmosphere processes to simulate biosignatures in a planetary systems science context (Olson et al., 2020; Reinhard et al., 2020).

The importance of environmental context. Oxygen-based biosignatures $\left(\mathrm{O}_{2}\right.$ and/or $\left.\mathrm{O}_{3}\right)$ are extremely promising, as they fulfill the three major requirements of a robust atmospheric biosignature: (1) reliability; (2) survivability; and (3) detectability. However, a number of potential "false positives" for $\mathrm{O}_{2} / \mathrm{O}_{3}$ biosignatures exist, rendering additional environmental context critical for interpreting oxygen-based biosignatures. For example, information about the host star (spectral type, age, activity level), major planet characteristics (size, orbit, mass), and accessory atmospheric species $\left(\mathrm{H}_{2} \mathrm{O}, \mathrm{CO}_{2}, \mathrm{CO}, \mathrm{CH}_{4}, \mathrm{~N}_{4}\right)$ can all help to diagnose pathological high- $\mathrm{O}_{2} / \mathrm{O}_{3}$ cases. Similarly, Earth's atmospheric evolution demonstrates that biogenic gases may remain at undetectable levels despite their production by a surface biosphere (Rugheimer and Kaltenegger, 2018).

Planetary characteristics that may enhance the likelihood of such "false positives" should be considered when selecting targets for biosignature searches. Careful selection of targets can help mitigate against the likelihood of false positive $\mathrm{O}_{2} / \mathrm{O}_{3}$ signals. For example, selection of older F, G, K or early M dwarf targets (M0-M3) would help guard against false positive $\mathrm{O}_{2} / \mathrm{O}_{3}$ signals associated with water loss, while potentially increasing the probability that biogenic $\mathrm{O}_{2} / \mathrm{O}_{3}$ will have accumulated to detectable levels. We suggest an integrated observation strategy for fingerprinting oxygenic photosynthetic biospheres on terrestrial planets with the following major steps: (1) planet detection and preliminary characterization; (2) search for $\mathrm{O}_{2} / \mathrm{O}_{3}$ spectral features with high-resolution spectroscopy; (3) further characterization and elimination of potential false positives; (4) detailed characterization and the search for secondary biosignatures. The identification of a pigment spectral feature would be a particularly complementary biosignature $\mathrm{O}_{2} / \mathrm{O}_{3}$ detection, because it would be consistent with the hypothesis that the $\mathrm{O}_{2}$ was generated by oxygenic photosynthesis. To further improve confidence in identifying surface signs of photosynthesis, the reflection spectra of the mineral background must also be characterized. Newly developed measurements such as the linear and circular polarization spectra of chiral biomolecules can potentially help rule out such false positives. In addition, models that address the surface coverage of a planet are needed to better understand the detectability of these signals.

\section{Scientific Progress in the Next 20 Years}

Cross-disciplinary quantitative frameworks. Much of the top-level theory of biosignatures is described in qualitative terms, and the associated advice to mission/instrument design teams is similarly qualitative. For example, we know that the confirmation of biosignatures 
requires a comprehensive classification of the planetary environment, which in turn leads to a suggestion to obtain observations with as broad of a wavelength range as possible. But evaluation of detailed trade-offs for specific instruments, and eventually the interpretation of data from biosignature searches, will be best enabled by a more quantitative framework.

A major challenge in such quantification is that assessing the presence or absence of life on a planet is an inherently complex problem, requiring comprehensive analyses of the planetary context. And a planet will have multiple systems that interact with each other, often in non-linear ways. Accounting for this in a quantified manner - and doing so in a way that is flexible enough to handle alien worlds with potentially alien climates and potentially alien life - requires an encompassing framework. At the EBWWW, a variety of approaches were discussed, including: process-based planetary system models; quantification of thermodynamic and/or kinetic disequilibrium in a planet's atmosphere (after Krissanssen- Totten et al., 2016); assessment of the complexity of atmospheric photochemical networks (after Holme et al. 2011); and utilization of Bayes' Theorem to assess the data from a single planet or a series of planets. Bayes' Theorem, in particular, was identified as having the potential to advance our field's ability to meaningfully evaluate sparse data, and as a framework for combining understanding from diverse scientific disciplines.

According to Bayes' Theorem, one can calculate the conditional probability that something is true, such as the likelihood of a system having a given property based on available data. An example mathematical formalism for exoplanet biosignatures is shown in Figure 1, from Catling et al. (2018). This derivation specifically dissects what might be observed ( $D=$ data) given either the presence or absence of life within a particular exoplanet environment context $(C=$ context), i.e., $P($ data|context and life) and $P($ data|context and no life $)$, respectively. The conditional probabilities here account for the intertwining of life with its environment, such that they cannot be independent. $P$ (life $\mid$ context) is a quantitative expression of likelihood of life given the context of the exoplanet,

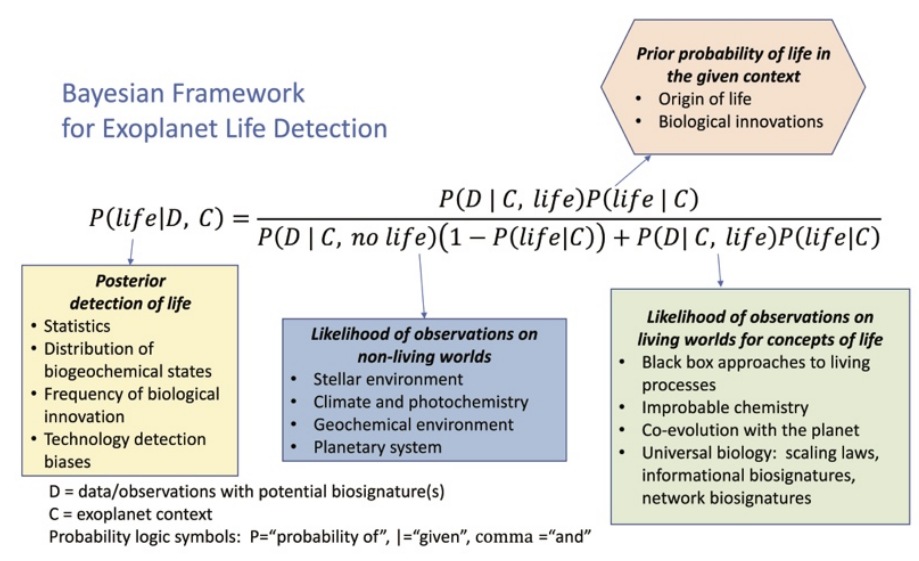

Figure 1. A Bayesian framework, applied to the detection of life on extrasolar planets. Equation from Catling, et al., 2018.

Adapted from Walker et al., 2018. such as amenability to habitability. This is distinct from $P$ (life) (the probability of life occurring at all in the universe). The latter might be estimated from how quickly life emerged on Earth, but is truly quantifiable only with large statistics, after more examples of life have been already discovered, which Walker et al. (2018) expand upon. Bayes' Theorem also provides a means to incorporate uncertainty in data (Parviainen, 2017), additional types and novel concepts of life, such as exotic adaptations, network theory, alternative chemistry, or statistics from ensemble investigations, and in general new data and ideas as they develop (e.g., Deeg et al., 2017). The Bayesian approach thus affords the synthesis of diverse areas of knowledge into a flexible, quantitative framework. It also is highly useful for identifying the terms most challenging to quantify. Given the highly interdisciplinary nature of the search for exoplanet 
biosignatures, adoption of a Bayesian concept is encouraged to help scientists work across disciplines, identify the significance of critical unknowns, and provide quantitative assessments of confidence in scientific conclusions.

The community is beginning to build comprehensive modeling tools, and the future research directions required to quantify our assessments are reviewed in the EBWWW paper by Walker et al. (2018). The tools for simulating data that could come from inhabited/uninhabited worlds are already under development with both flexible 1-dimensional atmospheric models that can be coupled to subsurface and escape models, and comprehensive but less flexible 3dimensional global climate models. Current work - often by large interdisciplinary teams - is increasing the comprehensiveness of the former models as well as the flexibility of the latter. This development of models must continue - and the community involvement in their development must be expanded. We also require advancements in chemistry and biology research on life's origins on Earth, and the environments in which life might originate elsewhere, to help with our assessments of $\mathrm{P}($ life). Finally, we must advance our grasp of the likelihood of certain biological innovations, and better understand the full range of metabolisms life can utilize for obtaining energy, beyond those found on modern-day Earth.

Many of these future advancements are an outgrowth from or have an analogy in the search for life inside the Solar System. Biosignatures via complexity were originally developed as a concept for life detection on Europa or other ocean worlds. The use of gas fluxes instead of gas concentrations could be useful in the search for present-day life on Mars or Titan (see white paper by Garvin et al. on "Mars Trace Gas Fluxes: Critical Strategies and Implications for the Upcoming Decade.") The attempts to develop comprehensive ecosystem models has its grounding in our studies of changes to Earth's biosphere over time. And the overall statistical/quantitative framework is something all of these communities are advocating. An additional advantage of this parallel push is that it will allow comparisons across these different searches, which would otherwise use different search strategies. It will also allow us to formally apply the results of one search to the others, for example in the form of priors.

\section{Telescopes in Planning or Development}

The most critical step in our search for extrasolar life is to detect spectroscopic properties of potentially habitable planets. A handful of Earth-sized planets in the HZs of late-type stars have already been identified (Anglada- Escud'e et al., 2016; Dittmann et al., 2017; Gillon et al., 2017), including a few that are close enough for follow-up observation. Soon, discoveries and astrophysical characterization of similar targets will be accelerated by TESS (2018-), CHEOPS (2018-), and ongoing/future ground- based RV surveys. Follow-up observations of such targets could be conducted by the James Webb Space Telescope JWST (2019-), and the next generation ground-based telescopes (GMT, TMT, ELT: 2020s-) and next-generation flagship space telescopes (OST, LUVOIR, HabEx) armed with high-resolution and/or high-contrast instruments. The detectability of the specific features depends on the system properties of the targets as well as the noise floor. And we note that the false positive concerns noted above (as well as concerns about habitability) are greatest for the stellar targets whose planets we will be able to see with this technique. Such concerns should not dissuade us from these observations, but they do make target selection and precursor observations of stellar host properties critical. The characterization of Earth-like HZ planets around Solar-type stars will require more sensitive observations. The PLATO (2026-) mission is specifically targeted at transiting planets in a wider parameter space, including small HZ planets around Solar-type stars. The spectroscopic characterization of 
potentially Earth-like worlds around Sun-like stars demands space-based high-contrast observations. These observations are not feasible with current and planned facilities, but are among the driving science goals for HabEx and LUVOIR.

These telescopes can be complementary to Solar System missions in multiple ways. Most directly, they will carry instrumentation that can provide tremendous advances in our observations of Solar System worlds. They could provide substantial increases in spectral and spatial resolution over current telescope platforms. They also enable longer observation timescales than can be obtained in fly-by and short-duration missions. This allows them to play a key support role for Solar System missions. For example, they could provide long-distance reconnaissance of ocean worlds, as a fly-by, orbiting, landing, or sample return mission approaches its target. This would help that target-specific mission to map geysers, local geomorphology, and other surface features or hazards in advance of final orbit determination, entry, descent, and landing. They also will use instruments/techniques that are common across many Solar System missions, in particular cameras and spectrographs. This creates shared incentives for some aspects of technology development, and enhances the potential for a broader community to engage in data analysis and planet characterization across both exoplanet and Solar System targets.

\section{Existing and Needed Partnerships}

The EBWWW revealed that the search for exoplanet life is still largely driven by astronomers, and that this field requires more input from origins of life researchers and biologists to advance a process-based understanding for planetary biosignatures indicative of extant life. This includes assessing the a priori likelihood that a planet may have life, or that life may have evolved in a given planet's past environment. These advances will require fundamental research into the origins of life and its many metabolic processes, in particular for environments that vary from those of the modern Earth. Thus, collaboration between astronomers and origin of life researchers, biologists, geochemists, and planetary scientists who investigate habitability and in situ biosignatures for extant or 'recently dead' life on Mars and ocean worlds is critical to defining research questions for exoplanets framed in an environmental context. Additionally, collaboration between the exoplanet community and researchers working in the mature field of in situ biosignatures of extinct life on the early Earth and Mars will be key for sharing insights gained and lessons learned from using multiple lines of evidence to demonstrate convincing biogenicity of putative biosignatures. All of these partnerships would therefore benefit searches for life, regardless of the target on which that search takes place. For a broader discussion of the potential for such partnerships, please see the white paper (Domagal-Goldman, et al., 2020) on "Astrobiology as a NASA Grand Challenge."

\section{Realizing NASA's life detection goals}

To realize our goals, and to enable probabilistic assessments of whether or not a planet has life, we require the following developments for exoplanet biosignature science, and identify synergies between exoplanet and Solar System life detection efforts:

- A more complete incorporation of biological expertise into exoplanet biosignature characterization.

- An improved capability to predict the expression of anoxygenic and oxygenic photosynthesis in different stellar-planetary environments.

- Evaluation of the wealth of potential new biosignatures, both surface and gaseous, and consideration of their potential false positives and false negatives. 
- Sustained institutional support to characterize the physical and chemical properties of biogenic volatile species.

- Models of fundamental abiotic processes under planetary conditions different than our own.

- Development and infrastructure support for 3-D general circulation models (GCMs) and models of ocean biogeochemistry for exoplanets, to simulate biosignatures in 3-D.

- Expanded coupling of 1-D planetary models for mantles, atmospheric chemistry, climate, ocean, biology, and atmospheric escape processes, with different stellar inputs to simulate biosignatures in a planet systems context.

- Improved accounting of model uncertainties.

- Utilize a Bayesian framework to foster integration of diverse scientific disciplines and to accommodate new data and novel concepts in order to enhance further development in the classroom and in collaborative research.

- Broad wavelength coverage by missions to ensure observation of the full planetary context.

- The diversity of potential biosignatures across targets requires community-level efforts be pursued to establish a standardized, evaluative framework that supports apples-to-apples assessment of their utility in addressing life detection objectives (Hoehler et al., 2020).

The Bayesian framework is critical as a quantitative approach that will advance our field in multiple ways. For exoplanet researchers, it will be a powerful way to consider future mission/instrument trade-offs, or to inform future target selection. For our peers who are searching for life on planets around other stars and at Mars and ocean worlds within our own Solar System, it will provide a comparative tool with different proposed biosignatures for other targets. For our scientific colleagues beyond exoplanets, it will provide a rigorous test of our conclusions. And for the general public and stakeholders, it will lead to the ability to clearly and consistently communicate our level of confidence that we are not alone.

\section{References}

Anglada-Escud'e, G., et al. (2016) Nature 536:437.; Arney G., et al. (2016) Astrobiology 16:873.; Catling D.C., et al. (2018) Astrobiology. arXiv:1705.06381; Deeg H. J., et al. (2017) Handbook of Exoplanets, Springer.; Des Marais D.J., et al. (2002) Astrobiology 2:153.; Dittmann, J.A., et al. (2017) Nature 544:333; Fujii Y., et al. (2018) Astrobiology. arXiv:1705.07098; Gillon M., et al. (2017) Nature 542:456; Hegde S., et al. (2015) PNAS 112:3886.; Ho M.Y., et al. (2016) Science 353:9178.; Holme P., et al. (2011) PLoS ONE 6:19759.; Krissanssen-Totton J., et al. (2016) Astrobiology, 16:39.; Li Y.Q., et al. (2015) Funct. Plant Biol. 42:493.; Luger R., et al. (2015) Astrobiology 15:119; ; Meadows V.S., et al. (2018) Astrobiology. arXiv:1705.07560; Parviainen H. (2017) In: Handbook of Exoplanets, Springer pp 1-24.; Reinhard C., et al. (2020) Geoscientific Model Development https://doi.org/10.5194/gmd-2020-32; Robinson, T.D., et al. (2011) Astrobiology 11:393,; Rugheimer and Keltenegger (2018) Astrophys. Journal. arXiv:1712.10027; Schwieterman E.W., et al. (2015a) Astrobiology 15:341.; Schwieterman, E.W., et al. (2015b) Astrophys. Journal 810:57.; Schwieterman E.W., et al. (2018) Astrobiology. arXiv:1705.05791; Seager S., et al. (2016) Astrobiology 16:465.; Takizawa K., et al. (2017) Nature Sci. Reports 7:id.7561; Turbet M., et al. (2016) Astron. Astrophys. 596.; Walker S.I., et al. (2018) Astrobiology. arXiv:1705.08071; Way M.J., et al. (2017) Astrophys. Journal Suppl. Series 231:12.; Wordsworth R., et al. (2014) Astrophys. Journal Letters, 785. 